\title{
Uma Nota Sobre o Papel da Credibilidade da Política Monetária e Fiscal: Evidências para o Brasil
}

Gabriel Caldas Montes*, Antonio Carlos de Jesus Assumpção ${ }^{\dagger}$

\author{
Conteúdo: 1. Introdução; 2. Breve Resenha da Literatura; 3. Análise Empírica; \\ 4. Estimativas; 5. Conclusão; A. Apêndice. \\ Palavras-chave: Reputação, Credibilidade, Taxa de Juros, Spread, Consumo. \\ Códigos JEL: $\quad$ E21, E43, E52, E62.
}

Este estudo analisa: (i) a influência das reputações do Banco Central do Brasil e do governo e da credibilidade de suas políticas sobre o comportamento da taxa básica de juros, e o impacto das medidas de gerenciamento da dívida pública e do cenário macroeconômico sobre a taxa de juros; (ii) a influência da política monetária e do ambiente macroeconômico sobre o comportamento do consumo das famílias pelo canal do crédito. Os resultados sugerem que os ganhos de credibilidade e reputação monetária e fiscal foram importantes para explicar a trajetória da taxa básica de juros e o spread de crédito, influenciando o comportamento do consumo das famílias.

This study examines (i) the influence of the reputations of the Central Bank of Brazil and the Government and the credibility of their policies on the path of the basic interest rate, and also the impact of public debt management policies and the macroeconomic scenario on the basic interest rate; (ii) the influence of monetary policy and the macroeconomic environment on the behavior of household consumption through the credit channel. The results suggest that gains of credibility and monetary and fiscal reputation were important to explain the path of the basic interest rate and the credit spread, affecting the behavior of household consumption.

\footnotetext{
*Universidade Federal Fluminense, Departamento de Economia, Rua Geraldo Martins, 23, Niterói, Rio de Janeiro, CEP: 24.220-380, e Pesquisador do CNPq, Brasil. Corresponding author, Phone: (21) 27117778 E-mail: gabrielmontesuff@yahoo.com. br

†Universidade Federal Fluminense, Departamento de Economia. Rua Geraldo Martins, 23, Niterói, Rio de Janeiro, CEP: 24.220-380, Brasil.
} 


\section{INTRODUÇÃO}

Desde a publicação dos trabalhos de Kydland e Prescott (1977) e Barro e Gordon (1983), diversos estudos foram elaborados com intuito de mensurar a credibilidade e a reputação, analisando como ambas são afetadas pela condução da política econômica e como afetam as políticas futuras, como também o desempenho da economia (por exemplo, Agénor e Taylor, 1993, Cecchetti e Krause, 2002, de Mendonça, 2007).

Com o desenvolvimento do debate regras versus discrição, surgem diferentes propostas de tecnologias de compromisso - dentre essas, o regime de metas para inflação. Atualmente, o regime de metas para inflação é adotado por diversos países. Ao conferir importância à credibilidade e ao desenvolvimento da reputação, o regime requer comprometimento da autoridade monetária com o seu principal objetivo de longo prazo: manter a inflação compatível com a meta estabelecida e estável.

De modo a tingir esse objetivo, é necessário não apenas uma política monetária comprometida com suas metas, mas também uma política fiscal. Nesse sentido, as políticas monetárias e fiscais devem atuar de maneiras coordenadas, pois, a utilização da política monetária para controlar a inflação, sem que as finanças públicas estejam equilibradas, pode implicar em elevadas taxas de juros (Mishkin, 2007).

Desde a adoção do regime de metas para inflação no Brasil, estudos vêm sendo elaborados com intuito de buscar evidências acerca da influência da credibilidade da política monetária e da reputação do banco central sobre a condução da política monetária e sobre o desempenho da economia brasileira (de Mendonça e de Guimarães e Souza, 2009, Montes e Bastos, 2014). Contudo, são escassas as evidências acerca dos efeitos da credibilidade e da reputação fiscal sobre a economia brasileira, e não há trabalhos acerca do impacto desses aspectos sobre o comportamento da taxa de juros.

Portanto, o presente estudo analisa

(i) a influência das reputações do Banco Central do Brasil e do governo e da credibilidade de suas políticas sobre o comportamento da taxa básica de juros, como também o impacto das medidas de gerenciamento da dívida pública e do cenário macroeconômico sobre a taxa de juros, e;

(ii) a influência da política monetária e do ambiente macroeconômico sobre o comportamento do consumo das famílias por meio do canal do crédito.

O presente estudo contribui com a literatura ao fornecer evidências de que as reputações e credibilidades (monetária e fiscal) são importantes para a determinação da taxa básica de juros na economia brasileira, e esta, por sua vez, afeta o consumo das famílias por meio do canal de crédito (spread de crédito).

Além desta Introdução, o trabalho encontra-se dividido da seguinte maneira. Na seção dois é apresentada uma breve resenha da literatura acerca dos assuntos abordados no estudo. A seção três apresenta os dados utilizados na análise e a metodologia. Na seção quatro é elaborada a análise empírica. Por último, na conclusão, são expostos os principais resultados obtidos.

\section{BREVE RESENHA DA LITERATURA}

Em relação aos estudos que buscam mensurar a credibilidade e a reputação e analisar seus efeitos sobre a economia brasileira, destacam-se os trabalhos elaborados por de Mendonça (2007), o qual apresenta um índice de credibilidade para a autoridade monetária, e de Mendonça e de Guimarães e Souza (2009), o qual busca mensurar a credibilidade e a reputação relacionada à condução da política monetária. Este último trabalho analisa, dentre uma série de índices de credibilidade e de reputação, quais deles são mais úteis na previsão de variações na taxa de juros a partir do ano 2000 para o caso brasileiro. $\mathrm{O}$ trabalho encontra evidências de que mais credibilidade exige menores variações nas taxas de juros para o controle da inflação e, também, taxas de juros menores para manter a inflação dentro da meta. Na mesma linha, Montes e Bastos (2014) encontram evidências de que a reputação do Banco 
Central do Brasil (BCB) é importante para a formação de credibilidade do regime de metas para inflação e que a credibilidade é importante para reduzir o esforço da autoridade monetária no gerenciamento diário da liquidez.

Por sua vez, a política fiscal deveria ser conduzida de maneira coordenada com a política monetária citepMishkin:2007, pois elevados déficits fiscais tendem a pressionar a taxa de juros, tornando custoso, sob o ponto de vista do produto, a obtenção da estabilidade de preços. Portanto, é possível argumentar que não apenas a credibilidade da política monetária e a reputação do banco central são importantes para determinar o comportamento da taxa de juros, mas também a reputação da autoridade fiscal e a credibilidade de suas políticas.

Os trabalhos de Calvo e Guidotti (1990), Giavazzi e Pagano (1990) e Giavazzi e Missale (2004) exploram outros fatores além da redução da dívida pública, como por exemplo, a estrutura de indexação da dívida e o prazo de maturidade, como fatores importantes para dar suporte a uma política monetária que possa manter a estabilidade de preços com baixas taxas de juros.

O trabalho de Giavazzi e Missale (2004) analisou a administração da dívida pública no Brasil. Os autores consideram a estabilização da razão dívida/PIB como o principal objetivo da política fiscal no Brasil. Entretanto, ressaltam a importância da redução do risco por meio de mudança na composição da dívida. Assim, recomendam que a maior parte da dívida pública brasileira seja constituída por títulos prefixados e, também, indexados aos índices de preços, e que seja reduzida a participação dos títulos indexados à taxa básica de juros e à taxa de câmbio.

O estudo elaborado por de Mendonça e da Silva (2008) reforça o argumento de Giavazzi e Missale (2004), e mostra que o bom gerenciamento da dívida pública impacta diretamente a credibilidade da política econômica e a condução da política monetária. Os autores sugerem que uma dívida pública menos indexada à taxa básica de juros faz com que a autoridade monetária tenha maior liberdade na busca pela estabilidade de preços, resultando assim em maior credibilidade e, com isso, taxas de juros menores.

O trabalho elaborado por de Mendonça e Machado (2013) contribui com a literatura ao apresentar um índice de credibilidade fiscal com base em expectativas do mercado acerca da dívida pública em relação ao PIB. O estudo apresenta evidências empíricas para o caso brasileiro de que a maior credibilidade fiscal permite ao governo colocar em prática as propostas de Giavazzi e Missale (2004) acerca da estrutura de composição da dívida pública.

No tocante a transmissão da política monetária para a economia, o canal do crédito é apontado como importante mecanismo de transmissão (Bernanke e Blinder, 1992, Bernanke e Gertler, 1995). Como sugerem Bernanke e Gertler (1995), a transmissão da política monetária pelo canal do crédito pode ser compreendida por meio de outros dois canais: o canal de empréstimos bancários (bank lending channel) e o canal de balanços (balance sheet channel).

Em relação aos estudos, utilizando dados agregados, que abordam a relação entre credibilidade do regime de metas para inflação e transmissão da política monetária por meio do canal do crédito no Brasil (em particular, o canal de empréstimos bancários), a literatura é escassa, podendo ser destacados os trabalhos de Montes e Machado (2013) e Montes e Peixoto (2014).

Montes e Machado (2013) encontram evidências que a oferta de crédito é estimulada com o aumento da credibilidade. Montes e Peixoto (2014) analisaram o impacto das ações do banco central e do ambiente macroeconômico sobre a percepção de risco dos bancos, e, a influência dessa percepção de risco dos bancos sobre o spread de crédito. Os resultados indicam que os bancos reagem à política monetária alterando o spread das operações de crédito.

Apesar dos avanços na literatura sobre transmissão da política monetária, existem lacunas a serem preenchidas, como por exemplo, em relação à influência da política monetária sobre o consumo das famílias por meio do canal do crédito em países em desenvolvimento adotando metas para inflação. Assim, o presente estudo busca contribuir com a literatura oferecendo algumas evidências. 


\section{ANÁLISE EMPÍRICA}

A análise empírica aborda a importância da credibilidade e da reputação das autoridades fiscal e monetária, como também do ambiente macroeconômico, sobre o comportamento da taxa básica de juros, e; o efeito da política monetária sobre o consumo das famílias pelo canal do crédito (spread de crédito).

\subsection{Metodologia}

A análise empírica utiliza o método de mínimos quadrados ordinários (MQO), e o método dos momentos generalizados (GMM). De acordo com Hansen (1982), uma das razões para utilizar o método GMM é que, enquanto o método MQO geralmente apresenta problemas de autocorrelação serial, heterocedasticidade e não linearidade, fatores comuns em séries temporais macroeconômicas, o GMM oferece estimadores consistentes para a regressão. ${ }^{1}$

\subsection{Descrição dos Dados}

O período analisado vai de janeiro de 2003 a dezembro de $2012 .^{2}$ As séries, mensais, utilizadas são as seguintes:

- Taxa Básica de Juros (Selic) - Taxa de juros mensal anualizada em pontos percentuais (série $\mathrm{n}^{0}$ 4189 do BCB).

- Hiato do produto (gap) - A Série para o hiato do produto (gap) é calculada pela diferença entre o PIB real efetivo e sua tendência de longo prazo (obtida pelo filtro Hodrick-Prescott). O produto efetivo é a série mensal do PIB nominal do Banco Central do Brasil (série $\mathrm{n}^{\circ} 4380$ do $\mathrm{BCB}$ ) deflacionada pelo IGP-M (série $\mathrm{n}^{\circ} 7448$ do $\mathrm{BCB}$ ). A série foi dessazonalizada por meio do método census X12 multiplicativo default.

- Variação da inflação (inflavar) - A série utiliza a inflação mensal medida pelo IPCA (série $\mathrm{n}^{\circ} 433$ do $\mathrm{BCB})$. A série foi anualizada e, posteriormente, foi calculada a sua diferença em relação ao período anterior.

- Índice de credibilidade do regime de metas para inflação (icbc) - O estudo utiliza o índice proposto em de Mendonça (2007). ${ }^{3}$ Para o cálculo do índice foi utilizada a série de expectativas de inflação

\footnotetext{
${ }^{1}$ Como apontado por Wooldridge (2001): "to obtain a more efficient estimator than two-stage least squares (or ordinary least squares), one must have overriding restrictions". O método GMM escolhe os coeficientes de forma que os resíduos sejam ortogonais aos instrumentos utilizados. Este teste é conhecido como teste de sobreidentificação. Em relação à técnica empregada na estimação por GMM, Cragg (1983) indicou que a análise de sobreidentificação possui um importante papel na seleção das variáveis instrumentais para melhorar a eficiência dos estimadores. Desta forma, com o objetivo de testar esta propriedade para a validade das restrições de sobreidentificação, a estatística $J$ é apresentada citepHansen:1982.

${ }^{2} \mathrm{~A}$ justificativa para utilização deste período se baseia na disponibilidade das séries, como também devido aos seguintes aspectos: o período é posterior à adoção do regime de metas para inflação e compreende a fase em que o Tesouro Nacional apresenta maior comprometimento com a sustentabilidade da dívida pública e com a realização de ajustes na estrutura de gerenciamento da dívida pública.

${ }^{3}$ Apesar de diferentes índices de credibilidade já terem sido propostos, o presente trabalho não pretende analisar a influência e o poder de cada índice sobre o principal instrumento de política monetária no Brasil - embora essa pesquisa seja importante. Assim, a opção de usar o índice proposto por de Mendonça (2007) se justifica com base nos seguintes argumentos: (i) o índice é reconhecido pela literatura internacional, sendo este índice utilizado em vários estudos aplicados, (ii) simplicidade de compreensão e de preparação, (iii) o índice capta as mudanças e flutuações na credibilidade de uma forma compatível com o regime de metas para inflação adotado no Brasil, ou seja, o índice utiliza bandas pré-determinadas de tolerância, e não bandas de tolerância ad-hoc, como proposto por outros índices, e (iv) o índice é suficientemente rigoroso e pune adequadamente os desvios das expectativas de inflação em relação à meta de inflação.
} 
acumulada para os próximos 12 meses, divulgada pelo Banco Central do Brasil. $O$ índice assume valor igual a 1 quando a inflação anual esperada $(E(\pi))$ for igual a meta de inflação e decresce a medida que as expectativas para a inflação se desviam da meta anunciada. $O$ índice assume valores entre 0 e 1 quando a expectativa de inflação se situa dentro dos limites mínimo e máximo $\left(\pi_{t}^{*}\right)$ estabelecidos para cada ano e caso exceda um desses limites, assume valor igual a 0 . Assim:

$$
i c b c=\left\{\begin{array}{ll}
1 & \text { se } E(\pi)=\pi_{t}^{c} \\
1-\frac{1}{\pi_{t}^{*}-\pi_{t}^{c}}\left[E(\pi)-\pi_{t}^{c}\right] & \text { se } \pi_{t M i n}^{*}<E(\pi)<\text { se } \pi_{t M a x}^{*} \\
0 & \text { se } E(\pi) \geq \operatorname{se} \pi_{t M a x}^{*} \text { ou } E(\pi) \leq \text { se } \pi_{t M i n}^{*}
\end{array}\right\}
$$

- Índice de credibilidade Fiscal (icf): O estudo utiliza o índice desenvolvido por de Mendonça e Machado (2013). O índice de credibilidade fiscal leva em conta a informação disponibilizada por meio do relatório Focus do Banco Central do Brasil sobre as expectativas do mercado para a relação dívida/PIB. $O$ índice considera como limites inferior e superior uma relação dívida/PIB ${ }^{4}$ de $40 \%$ e $60 \%$ respectivamente. Tais limites foram estabelecidos no Tratado de Maastricht e, de acordo com o IMF - International Monetary Fund (2002), fornecem pontos de referência úteis, tendo em visto que uma razão dívida/PIB maior que esta referência aumenta a probabilidade de uma crise da dívida. Assim, o icf é calculado da seguinte maneira:

$$
i c f=\left\{\begin{array}{ll}
1 & \text { se } E_{t}\left(\text { debt }_{t+12} \leq d e b t^{\text {Min }}\right. \\
1-\left[\frac{\left[E_{t}\left(d e b t_{t+12}\right)-d e b t^{M i n}\right]}{d e b t_{t}^{\text {Max }}-d e b t_{t}^{\text {Min }}}\right] & \text { se } \pi_{t M i n}^{*}<E_{t}\left(\text { debt }_{t+12}\right)<d e b t^{M a x} \\
0 & \text { se } E_{t}\left(\text { debt }_{t+12}\right) \geq d e b t^{M a x}
\end{array}\right\}
$$

Logo, quando as expectativas de mercado acerca da relação dívida/PIB para os 12 meses seguintes são inferiores a 40\%, o risco de desequilíbrio fiscal é nulo e, portanto, o índice de credibilidade é igual a 1. Caso as expectativas superem o valor de $60 \%$, existe uma elevada probabilidade de desequilíbrio fiscal e, portanto, o índice de credibilidade é igual a 0 . Caso as expectativas se situem entre $40 \%$ e $60 \%$, o índice de credibilidade varia entre 0 e 1.

- Índice de reputação da Autoridade Monetária (irm) - É utilizado o índice desenvolvido por Montes e Bastos (2014). O índice de reputação utiliza a inflação observada anualizada, medida pelo IPCA, e é ponderada pelo hiato do produto. Assim,

$$
\begin{aligned}
& \operatorname{irm}=\left\{\begin{array}{ll}
1 & \text { se } \pi_{t M i n}^{*} \leq_{t O B S} \leq \pi_{t M a x}^{*} \\
1-\frac{1}{0.2-\pi_{t<a x}^{*}}\left[\pi_{O B S}-\pi_{t M a x}^{*}\right] & \text { se } \pi_{t M a x}^{*}<\pi_{O B S}<0.2 \\
1-\frac{1}{-\pi_{t M i n}^{*}}\left[\pi_{O B S}-\pi_{t M i n}^{*}\right] & \text { se } 0 \%<\pi_{O B S}<\pi_{t M i n}^{*} \\
0 & \text { se } \pi_{O B S} \geq 0.2 \text { ou } \pi_{O B S} \leq 0
\end{array}\right\} \\
&\left\{\begin{array}{ll}
\frac{Y_{n}}{Y} \quad \text { se } \frac{Y_{n}}{Y}<1 \\
\\
1 & \text { se } \frac{Y_{n}}{Y}>1
\end{array}\right\}
\end{aligned}
$$

A série do PIB utilizada foi obtida junto ao BCB (PIB acumulado nos últimos 12 meses - valorizado pelo IGP-DI centrado no mês, em R milhões), e sua tendência de longo prazo $\left(Y_{n}\right)$ foi obtida por meio do filtro de Hodrick-Prescott.

\footnotetext{
${ }^{4} \mathrm{~A}$ relação dívida/PIB refere-se à relação entre a dívida líquida do setor público e o PIB.
} 
- Índice de reputação fiscal (irf) - É utilizado o índice desenvolvido por Montes e Machado (2014). A série para o irf foi calculada utilizando os dados para a relação dívida/PIB (série 4513 do BCB), sendo o limite máximo de 60\%, estabelecido no Tratado de Maastricht, e o limite mínimo de $40 \%$, de acordo com IMF - International Monetary Fund (2002), pois fornecem pontos de referência úteis, tendo em visto que uma razão dívida/PIB maior que esta referência aumenta a probabilidade de uma crise da dívida. $\mathrm{O}$ irf é calculado da seguinte maneira:

$$
\operatorname{irf}=\left\{\begin{array}{ll}
1 & \text { se } d l s p \leq 0.4 \\
1-\frac{1}{0.2}(d l s p-0.4) & \text { se } 0.4<d l s p<0.6 \\
0 & \text { se } d l s p \geq 0.6
\end{array}\right\}
$$

- Indexador da Dívida Pública (index): Esta série foi construída por meio da razão entre dois índices: index2/index1. A série index2 representa o somatório da posição em carteira da participação dos seguintes indexadores da Dívida Pública brasileira: dívida pública indexada à Taxa de Juros Over/Selic (série $n^{0} 4177$ do BCB) e dívida pública indexada ao Câmbio (série $n^{0} 4173$ do BCB). A série index1 representa o somatório da posição em carteira da participação dos seguintes indexadores da Dívida Pública brasileira: dívida pública pré-fixada (série $\mathrm{n}^{\circ} 4178$ do $\mathrm{BCB}$ ), dívida pública indexada ao IPCA (série $\mathrm{n}^{\circ} 12001$ do BCB), dívida pública indexada ao IGP-M (série $\mathrm{n}^{\circ} 4175$ do $\mathrm{BCB}$ ) e dívida pública indexada ao IGP-DI (série $\mathrm{n}^{\circ} 4176$ do $\mathrm{BCB}$ ). Portanto, seguindo a ideia de Giavazzi e Missale (2004) de que a maior parte da dívida pública brasileira deveria ser constituída por títulos prefixados e por títulos indexados aos índices de preços e, por sua vez, as emissões de títulos indexados à taxa básica de juros e à taxa de câmbio deveriam ser reduzidas, a série index representa uma proxy para a falta de eficiência na administração da dívida pública no que diz respeito aos seus indexadores.

- Prazo da Dívida Pública (prz) - Representa o prazo médio de vencimento da dívida líquida do setor público em meses (série $\mathrm{n}^{\circ} 10621$ do $\mathrm{BCB}$ ). Esta série é utilizada seguindo as ideias de Giavazzi e Pagano (1990) e de Calvo e Guidotti (1990).

- Spread de Crédito (spread) - Série de spread médio cobrado nas operações de crédito com recursos livres em pontos percentuais (série $\mathrm{n}^{0} 3955$ do $\mathrm{BCB}$ ).

- Requerimento de Reservas (rreq) - Este instrumento de política monetária tem um impacto direto sobre o volume de crédito. A maneira de construir a série e os dados utilizados seguem Montes e Peixoto (2014) (séries 1883, 1884, 1886 e 17633 do BCB).

- Índice de Preços do Mercado Acionário (ibov) - A série do IBOVESPA foi obtida junto ao Banco Central do Brasil (série $n^{0}$ 7845). De acordo com Auel e de Mendonça (2011), essa variável representa uma proxy para o comportamento dos preços dos ativos no mercado financeiro nacional. A valorização dos ativos aumenta o poder das empresas, oferecendo uma maior garantia para o sistema financeiro ofertante de crédito.

- Taxa de inadimplência (default) - A taxa de inadimplência representa o nível de perdas sofridas pelos bancos em suas operações de crédito (série 13673 do BCB).

- Taxa de Câmbio (exch) - Taxa de câmbio R\$/US\$, média do período (série 3696 do BCB). A taxa de câmbio afeta o financiamento externo dos bancos e também o balanço dos bancos e empresas com dívidas em moeda estrangeira. Uma desvalorização cambial resulta em agravamento nas condições do mercado de crédito e, portanto, em aumento do spread de crédito. 
- Consumo das Famílias (conssa) - Série encadeada do índice de volume trimestral com ajuste sazonal, fornecida pelo IBGE. Para a obtenção da série mensal do consumo das famílias, os dados originais foram interpolados linearmente por meio do software e-views 7.0. ${ }^{5}$

- Taxa Real de Juros (spread_selic) - Taxa real de juros para o tomador final (famílias). Variável obtida pela soma entre a taxa de captação dos bancos, a taxa Selic (série 4198 do BCB) e o spread médio cobrado nas operações de crédito com recursos livres em pontos percentuais (série 3955 do $\mathrm{BCB}$ ), deflacionada pelo índice de preços ao consumidor amplo (série 433 do BCB). Como o spread está medido em pontos percentuais acima da taxa Selic, ao somar as séries Selic e spread e, posteriormente, deflacionar, obtém-se a taxa real de juros incidente sobre as decisões de consumo das famílias.

- Renda Real (rrer) - Série de renda real efetivamente recebida pelo trabalhador no emprego principal (obtida da Pesquisa Mensal de Emprego do IBGE). A série foi transformada, calculando-se a variação em relação ao mesmo período do ano anterior.

- Crédito (cred pib) - Série obtida junto ao Banco Central do Brasil (série $\mathrm{n}^{0} 17473$ ) e representa o saldo das operações de crédito ao setor privado em relação ao PIB.

- Renda Esperada (expcresc) - Como proxy para a renda esperada foi utilizada a expectativa de crescimento do PIB para o ano, divulgada pelo Banco Central do Brasil.

- Confiança do Consumidor (icc) - A confiança do consumidor é mensurada por meio do índice de confiança do consumidor, calculado pela Fecomércio.

\section{ESTIMATIVAS}

A primeira condição a ser testada antes de aplicar a análise de regressão é verificar se as séries não possuem raiz unitária, ou seja, se são estacionárias. Assim, foram aplicados os testes ADF (Augmented Dickey-Fuller), PP (Phillips-Perron) e KPSS (Kwiatkowski-Phillips-Schmidt-Shin). Os testes estão reportados nas tabelas A.1, A.2 e A.3 no Apêndice. ${ }^{6}$

Cabe ressaltar que todas as equações foram estimadas utilizando o estimador de Newey-West para corrigir problemas de autocorrelação e heteroscedasticidade. ${ }^{7}$ É importante esclarecer que a escolha dos lags foi determinada por meio da metodologia "geral para o específico" e obedecendo ao princípio da parcimônia.

\footnotetext{
${ }^{5} \mathrm{~A}$ opção pela interpolação da série trimestral dos gastos em consumo das famílias deve-se ao fato de que a série mensal de consumo disponível para a economia brasileira refere-se à produção de bens de consumo. Desta forma, a série mensal de consumo disponível difere da série de gastos em consumo das famílias na medida da variação de estoques.

${ }^{6} \mathrm{O}$ critério de escolha para determinar se a série é ou não estacionária combina os três testes da seguinte forma: para ser considerada estacionária a série deve passar no teste KPSS e passar em um dos dois outros testes, ADF ou PP. A vantagem de contrastar os resultados dos testes ADF e PP com o resultado do teste KPSS reside no baixo poder dos dois primeiros testes, que tendem a não rejeitar a hipótese nula demasiadas vezes, como destacam Franses e Haldrup (1994) e Cati et alii (1999). Ademais, Kwiatkowski et alii (1992) argumentam que o teste KPSS pode distinguir séries que aparentam ser estacionárias, séries que aparentam ter uma raiz unitária, e séries para as quais os dados não são suficientemente informativos para ter a certeza se são estacionárias ou integradas - como é o caso de séries com curta periodicidade.

${ }^{7}$ A presença de autocorrelação foi testada por meio do teste de Breusch-Godfrey. A presença de heteroscedasticidade foi testada por meio do teste ARCH. Ademais, nas estimações por MQO foi utilizado o teste RESET de Ramsey para detectar possíveis erros de especificação e nas estimações por GMM foi utilizada a estatística $J$.
} 


\subsection{Estimações relacionadas à Taxa Básica de Juros}

As estimações relacionadas à taxa básica de juros (Selic) foram realizadas considerando as variáveis de reputação (monetária e fiscal) e, as variáveis de credibilidade (monetária e fiscal). Entretanto, os efeitos da credibilidade (variável forward-looking) e da reputação (variável backward-looking) são estimados separadamente.

A equação geral a ser estimada para explicar o comportamento da taxa básica de juros, utilizando o conceito de reputação, é a seguinte:

$$
\begin{aligned}
\text { selic } & =\beta_{0}+\beta_{1} \operatorname{selic}_{(-1)}^{(+)}+\beta_{2} \operatorname{inflavar}_{(-2)}^{(+)}+\beta_{3} \operatorname{gap}_{(-3)}^{(+)}+\beta_{4} \operatorname{dindex}_{(-3)}^{(+)}+\beta_{5} \operatorname{dpr} z_{(-3)}^{(-)} \\
& +\beta_{6} \operatorname{irf}_{(-3)}^{(-)}+\beta_{7} d_{-} \operatorname{irm}_{(-1)}^{(-)}+u_{t}
\end{aligned}
$$

O sinal acima da variável refere-se ao sinal esperado da primeira derivada conforme prevê a teoria econômica e, $u_{t}$ representa o termo de erro aleatório (ruído branco).

Por sua vez, a equação geral a ser estimada para explicar o comportamento da taxa básica de juros, utilizando a credibilidade, é a seguinte:

$$
\begin{aligned}
\text { selic } & =\beta_{8}+\beta_{9} \operatorname{selic}_{(-1)}^{(+)}+\beta_{10} \operatorname{inflavar}_{(-1)}^{(+)}+\beta_{11} \operatorname{gap}_{(-1)}^{(+)}+\beta_{12} \operatorname{dindex}_{(-1)}^{(+)}+\beta_{13} d_{-} \text {pr } z_{(-1)}^{(-)} \\
& +\beta_{14} i c b c_{(-2)}^{(-)}+\beta_{15} d_{-} i c f_{(-1)}^{(-)}+\varepsilon_{t}
\end{aligned}
$$

O sinal acima da variável refere-se ao sinal esperado da primeira derivada conforme prevê a teoria econômica e, $\varepsilon_{t}$ representa o termo de erro aleatório (ruído branco).

As Tabelas 1 e 2 apresentam os resultados das estimativas por MQO e GMM.

Considerando os resultados das estimações das equações 1.3 e 2.3 , tanto no caso de OLS quanto GMM, os coeficientes estimados para a influência da inflação e da atividade econômica apresentam os sinais esperados e significância estatística. Portanto, a variação positiva da taxa de inflação e o aquecimento da economia medido pelo aumento no hiato do produto possuem relação positiva com a taxa básica de juros.

Os resultados das estimações das equações 1.3 e 2.3, tanto no caso de OLS quanto GMM, apontam que uma piora na estrutura de indexação da dívida pública (aumento na variável index) possui uma relação positiva com a taxa básica de juros. Por sua vez, a maturidade da dívida pública possui uma relação negativa com a taxa básica de juros. Esses resultados reforçam os argumentos de Calvo e Guidotti (1990), Giavazzi e Pagano (1990) e Giavazzi e Missale (2004).

Os resultados das estimações da equação 1.3, tanto no caso de OLS quanto GMM, apontam que a maior reputação da autoridade fiscal e a maior reputação da autoridade monetária tornam possível reduzir a taxa selic e, portanto, possuem efeito negativo sobre a taxa básica de juros. Por seu turno, os resultados das estimações da equação 2.3 , apontam que a maior credibilidade da autoridade fiscal e a maior credibilidade da autoridade monetária tornam possível reduzir a taxa selic e, portanto, possuem efeito negativo sobre a taxa básica de juros. Assim, os resultados apontam que quando aumenta o comprometimento das autoridades monetária e fiscal com seus objetivos, aumentando as reputações e credibilidades monetária e fiscal, a autoridade monetária ganha graus de liberdade para reduzir a taxa básica de juros. 
Tabela 1: Estimações por MQO e GMM para Taxa de Juros - Reputação

\begin{tabular}{|c|c|c|c|c|c|c|}
\hline \multirow[t]{2}{*}{ Variáveis Explicativas } & \multicolumn{3}{|c|}{ OLS } & \multicolumn{3}{|c|}{ GMM } \\
\hline & Equação (1.1) & Equação (1.2) & Equação (1.3) & Equação (1.1) & Equação (1.2) & Equação (1.3) \\
\hline \multirow[t]{3}{*}{ C } & 0.2389 & 0.0873 & $1.0944^{* *}$ & 0.0528 & 0.3163 & $1.1607^{* *}$ \\
\hline & $(0.2497)$ & $(0.1937)$ & $(0.5008)$ & $(0.2631)$ & $(0.2310)$ & $(0.4766)$ \\
\hline & [0.9571] & [0.4509] & [2.1851] & [0.2006] & [1.3691] & [2.4353] \\
\hline \multirow[t]{3}{*}{ SELIC(-1) } & $0.9721^{* * *}$ & $0.9896^{* * *}$ & $0.9565^{* * *}$ & $0.9871^{* * *}$ & $0.9799^{* * *}$ & $0.9558^{* * *}$ \\
\hline & $(0.0208)$ & $(0.0149)$ & $(0.0228)$ & $(0.0222)$ & $(0.0176)$ & $(0.0233)$ \\
\hline & [46.7972] & [66.3514] & [41.9190] & [44.3883] & [55.5264] & [40.9389] \\
\hline \multirow[t]{3}{*}{ INFLAVAR(-2) } & $0.2888^{* *}$ & 0.1449 & $0.1605^{* *}$ & $0.5126^{*}$ & -0.0246 & $0.2007^{*}$ \\
\hline & $(0.1292)$ & (0.1249) & $(0.0773)$ & $(0.2697)$ & $(0.1669)$ & $(0.1074)$ \\
\hline & [2.2359] & [1.1612] & {$[2.0764]$} & [1.9009] & {$[-0.1472]$} & [1.8691] \\
\hline \multirow[t]{3}{*}{ GAP(-3) } & $0.0236^{* * *}$ & $0.0275^{* * *}$ & $0.0239^{* * *}$ & $0.0223^{* *}$ & $0.0485^{* * *}$ & $0.0374^{* * *}$ \\
\hline & $(0.0079)$ & $(0.0083)$ & $(0.0071)$ & $(0.0109)$ & $(0.0126)$ & $(0.0107)$ \\
\hline & {$[2.9707]$} & [3.3148] & {$[3.3728]$} & {$[2.0558]$} & [3.8409] & [3.5108] \\
\hline \multirow[t]{3}{*}{ D_INDEX(-3) } & & $1.8732^{* * *}$ & $1.7897^{* * *}$ & & $3.0906^{* * *}$ & $2.3829^{*}$ \\
\hline & & $(0.7024)$ & $(0.6578)$ & & $(0.9092)$ & (1.2536) \\
\hline & & [2.6669] & {$[2.7208]$} & & [3.3990] & [1.9008] \\
\hline \multirow[t]{3}{*}{ D_PRZ(-3) } & & $-0.0973^{* *}$ & $-0.0949^{* *}$ & & $-0.6531^{* * *}$ & $-0.5206^{* * *}$ \\
\hline & & $(0.0422)$ & $(0.0373)$ & & $(0.1923)$ & $(0.1632)$ \\
\hline & & {$[-2.3040]$} & {$[-2.5453]$} & & {$[-3.3958]$} & {$[-3.1894]$} \\
\hline \multirow[t]{3}{*}{$\operatorname{IRF}(-3)$} & & & $-0.7484^{* *}$ & & & $-0.7275^{* *}$ \\
\hline & & & $(0.3045)$ & & & $(0.2965)$ \\
\hline & & & {$[-2.4581]$} & & & {$[-2.4535]$} \\
\hline \multirow[t]{3}{*}{ D IRM(-1) } & & & $-3.4090^{* * *}$ & & & $-4.2086^{*}$ \\
\hline & & & $(1.2836)$ & & & (2.4579) \\
\hline & & & {$[-2.6557]$} & & & {$[-1.7123]$} \\
\hline$R^{2}$ & 0,9912 & 0,9919 & 0,9933 & 0,9886 & 0,9770 & 0,9836 \\
\hline Adjusted $R^{2}$ & 0,9909 & 0,9915 & 0,9929 & 0,9883 & 0,9759 & 0,9825 \\
\hline$F$-statistic & 4219,4010 & 2679,0780 & 2283,7180 & & & \\
\hline Prob.(F-statistic) & 0,0000 & 0,0000 & 0,0000 & & & \\
\hline$J$-statistic & & & & 7,4977 & 7,1438 & 9,8274 \\
\hline Prob.( $J$-statistic) & & & & 0,0576 & 0,3078 & 0,2774 \\
\hline Ramsey RESET (1) & 2,5992 & 1,9712 & 1,5880 & & & \\
\hline Prob.(Ramsey RESET) & 0,0106 & 0,0512 & 0,1152 & & & \\
\hline Heteroskedasticity ARCH (1) & 81,9281 & 9,5270 & 4,8968 & & & \\
\hline Prob.(Heteroskedasticity) & 0,0000 & 0,0025 & 0,0289 & & & \\
\hline Breusch-Godfrey - LM Test (1) & 177,5784 & 94,1848 & 75,1724 & & & \\
\hline Prob.(Breusch-Godfrey - LM Test) & 0,0000 & 0,0000 & 0,0000 & & & \\
\hline \multicolumn{7}{|l|}{ GMM - instrumentos } \\
\hline selic(-2 to -3$)$ inflavar(- -3 to -4$)$ gap(- -4 to & $\operatorname{dex}(-4$ to -6$)$ & $(-4$ to -5$)$ irf(- -4 & d_irmbal(-2 to -3$)$ & & & \\
\hline
\end{tabular}


Tabela 2: Estimações por MQO e GMM para Taxa de Juros - Credibilidade

\begin{tabular}{|c|c|c|c|c|c|c|}
\hline \multirow[t]{2}{*}{ Variáveis Explicativas } & \multicolumn{3}{|c|}{ OLS } & \multicolumn{3}{|c|}{ GMM } \\
\hline & Equação (2.1) & Equação (2.2) & Equação (2.3) & Equação (2.1) & Equação (2.2) & Equação (2.3) \\
\hline \multirow[t]{3}{*}{ C } & 0.1249 & -0.1191 & 0.2133 & -0.1559 & -0.1864 & 0.0316 \\
\hline & $(0.2328)$ & $(0.1378)$ & $(0.2436)$ & $(0.2388)$ & $(0.1297)$ & $(0.2056)$ \\
\hline & {$[0.5365]$} & {$[-0.8643]$} & {$[0.8757]$} & {$[-0.6533]$} & {$[-1.4374]$} & {$[0.1537]$} \\
\hline \multirow[t]{3}{*}{ SELIC(-1) } & $0.9816^{* * *}$ & $1.0067^{* * *}$ & $1.0001^{* * *}$ & $1.0028^{* * *}$ & $1.0158^{* * *}$ & $1.0301^{* * *}$ \\
\hline & $(0.0192)$ & $(0.0106)$ & $(0.0114)$ & $(0.0201)$ & $(0.0104)$ & $(0.0142)$ \\
\hline & [51.1451] & [94.5338] & [87.6173] & [49.9238] & [97.7577] & [72.2495] \\
\hline \multirow[t]{3}{*}{$\operatorname{INFLAVAR}(-1)$} & $0.3802^{* * *}$ & $0.2915^{* * *}$ & $0.2370^{* *}$ & $0.7468^{* * *}$ & 0.1929 & $0.2710^{*}$ \\
\hline & $(0.0937)$ & $(0.0934)$ & $(0.0913)$ & $(0.2267)$ & $(0.2075)$ & $(0.1517)$ \\
\hline & {$[4.0584]$} & {$[3.1218]$} & {$[2.5958]$} & {$[3.2948]$} & [0.9295] & [1.7859] \\
\hline \multirow[t]{3}{*}{ GAP(-1) } & $0.0172^{* *}$ & $0.0218^{* *}$ & $0.0235^{* * *}$ & $0.0203^{* *}$ & $0.0364^{* * * *}$ & $0.0395^{* * * *}$ \\
\hline & $(0.0084)$ & $(0.0083)$ & $(0.0076)$ & $(0.0091)$ & $(0.0086)$ & $(0.0075)$ \\
\hline & {$[2.0352]$} & [2.6153] & [3.1021] & {$[2.2429]$} & {$[4.2239]$} & [5.2585] \\
\hline \multirow[t]{3}{*}{ D_INDEX(-1) } & & $2.0749^{* * *}$ & $2.1900^{* * * *}$ & & $3.9364^{* * *}$ & $3.5184^{* * *}$ \\
\hline & & $(0.6991)$ & $(0.6886)$ & & $(0.9947)$ & $(0.7821)$ \\
\hline & & {$[2.9678]$} & [3.1801] & & [3.9573] & {$[4.4986]$} \\
\hline \multirow[t]{3}{*}{ D_PRZ(-1) } & & $-0.1001^{* *}$ & $-0.0878^{* *}$ & & $-0.2475^{* *}$ & $-0.2426^{* *}$ \\
\hline & & $(0.0409)$ & $(0.0396)$ & & $(0.1222)$ & $(0.1091)$ \\
\hline & & {$[-2.4480]$} & {$[-2.2170]$} & & {$[-2.0259]$} & {$[-2.2229]$} \\
\hline \multirow[t]{3}{*}{$\operatorname{ICBC}(-2)$} & & & $-0.3718^{*}$ & & & $-0.4061^{* *}$ \\
\hline & & & $(0.1937)$ & & & $(0.1841)$ \\
\hline & & & {$[-1.9192]$} & & & {$[-2.2055]$} \\
\hline \multirow[t]{3}{*}{ D_ICF(-1) } & & & -1.2784 & & & $-19.0211^{* *}$ \\
\hline & & & $(4.5937)$ & & & (7.9095) \\
\hline & & & {$[-0.2783]$} & & & {$[-2.4048]$} \\
\hline$R^{2}$ & 0,9916 & 0,9931 & 0,9934 & 0,9880 & 0,9896 & 0,9875 \\
\hline Adjusted $R^{2}$ & 0,9914 & 0,9928 & 0,9930 & 0,9877 & 0,9891 & 0,9867 \\
\hline$F$-statistic & 4511,2670 & 3223,8720 & 2380,0770 & & & \\
\hline Prob.(F-statistic) & 0,0000 & 0,0000 & 0,0000 & & & \\
\hline$J$-statistic & & & & 7,5670 & 13,0657 & 12,1903 \\
\hline Prob.( $J$-statistic) & & & & 0,0559 & 0,1597 & 0,4305 \\
\hline Ramsey RESET (1) & 2,0385 & 0,6067 & 1,3922 & & & \\
\hline Prob.(Ramsey RESET) & 0,0438 & 0,5453 & 0,1667 & & & \\
\hline Heteroskedasticity ARCH (1) & 46,2399 & 5,7025 & 4,9045 & & & \\
\hline Prob.(Heteroskedasticity) & 0,0000 & 0,0186 & 0,0288 & & & \\
\hline Breusch-Godfrey - LM Test (1) & 138,3158 & 62,8015 & 56,7250 & & & \\
\hline Prob.(Breusch-Godfrey - LM Test) & 0,0000 & 0,0000 & 0,0000 & & & \\
\hline
\end{tabular}

GMM - instrumentos

selic(-2 to -3) inflavar(-2 to -3) gap(-2 to -3) d_ index(-2 to -5) d_prz(-2 to -5) icbc(-3 to -4$) d_{-}$icf(-2 to -4$)$

Níveis de significância: ***denota $1 \%,{ }^{* *}$ denota $5 \%$ e *denota $10 \%$. Erros padrão entre parênteses e estatística $t$ entre colchetes.

Fonte: Estimações elaboradas pelos autores. 


\subsection{Estimação do spread de Crédito}

As equações estimadas para explicar o comportamento do spread de crédito são apresentadas abaixo (o sinal acima da variável refere-se ao sinal esperado da primeira derivada) e, $\delta_{t}$ representa o termo de erro aleatório (ruído branco).

$$
\begin{aligned}
\text { spread } & \alpha_{0}+\alpha_{1} \text { spread }_{(-1)}^{(+)}+\alpha_{2} \text { selic }_{(-1)}^{(+)}+\alpha_{3} d_{-} \text {rreq }_{(-3)}^{(+)}+\alpha_{4} d_{-} \text {default }_{(-2)}^{(+)}+\delta_{t} \\
\text { spread } & =\alpha_{5}+\alpha_{6} \operatorname{spread}_{(-1)}^{(+)}+\alpha_{7} \operatorname{selic}_{(-1)}^{(+)}+\alpha_{8} d_{-} \text {rreq }_{(-3)}^{(+)}+\alpha_{9} d_{-} \text {default }_{(-2)}^{(+)} \\
& +\alpha_{10} d_{-} \operatorname{exch}_{(-2)}^{(+)}+\delta_{t}^{i} \\
\text { spread } & =\alpha_{11}+\alpha_{12} \operatorname{spread}_{(-1)}^{(+)}+\alpha_{13} \operatorname{selic}_{(-1)}^{(+)}+\alpha_{14} d_{-} r r e q_{(-3)}^{(+)}+\alpha_{15} d_{-} \text {default }_{(-2)}^{(+)} \\
& +\alpha_{16} d_{-} \text {ibov }_{(-2)}^{(-)}+\delta_{t}^{i i}
\end{aligned}
$$

A Tabela 3 apresenta os resultados das estimativas por MQO e GMM. Considerando os resultados das estimações, os coeficientes estimados apresentam os sinais esperados e significância estatística, por MQO e GMM.

No tocante às estimações das equações (3.2) e (3.3), as evidências apontam que a taxa básica de juros, o volume de reservas requeridas (compulsório) e a inadimplência possuem uma relação positiva com o spread de crédito. Esses achados corroboram os resultados encontrados nos trabalhos de Auel e de Mendonça (2011) e Montes e Peixoto (2014).

A equação (3.2) considera o efeito exercido pela taxa de câmbio sobre o spread de crédito, o resultado sugere a existência de uma relação positiva entre as duas variáveis. A equação (3.3) considera o efeito exercido pelo IBOVESPA sobre o spread de crédito, o resultado sugere a existência de uma relação negativa entre as duas variáveis. Ambos os resultados também foram obtidos por Auel e de Mendonça (2011).

Portanto, os resultados encontrados corroboram aqueles já existentes na literatura acerca dos efeitos das políticas monetária sobre o spread de crédito e, em particular, sugerem que, as reduções observadas na taxa básica de juros tiveram influência sobre a redução do spread de crédito no Brasil, para o período analisado. 
Tabela 3: Estimações por MQO e GMM para o spread

\begin{tabular}{|c|c|c|c|c|c|c|}
\hline \multirow[t]{2}{*}{ Variáveis Explicativas } & \multicolumn{3}{|c|}{ OLS } & \multicolumn{3}{|c|}{ GMM } \\
\hline & Equação (3.1) & Equação (3.2) & Equação (3.3) & Equação (3.1) & Equação (3.2) & Equação (3.3) \\
\hline \multirow[t]{3}{*}{ c } & $2.7832^{* * *}$ & $2.7799^{* * *}$ & $2.6626^{* * *}$ & $2.2165^{* * *}$ & $1.8465^{* * *}$ & $1.2214^{* *}$ \\
\hline & $(0.9616)$ & (0.9132) & $(0.9531)$ & (0.4969) & (0.5556) & (0.5789) \\
\hline & {$[2.8942]$} & [3.0443] & [2.7937] & [4.4603] & {$[3.3236]$} & [2.1096] \\
\hline \multirow[t]{3}{*}{ SPREAD(-1) } & $0.8619^{* * *}$ & $0.8532^{* * *}$ & $0.8687^{* * *}$ & $0.8680^{* * *}$ & $0.8818^{* * *}$ & $0.9037^{* * * *}$ \\
\hline & $(0.0393)$ & $(0.0342)$ & $(0.0360)$ & $(0.0216)$ & $(0.0208)$ & $(0.0249)$ \\
\hline & [21.9308] & [24.9801] & [24.0952] & [40.0988] & [42.3368] & [36.2191] \\
\hline \multirow[t]{3}{*}{ SELIC(-1) } & $0.1313^{* * *}$ & $0.1571^{* * *}$ & $0.1253^{* * *}$ & $0.1581^{* * *}$ & $0.1534^{* * *}$ & $0.1402^{* * *}$ \\
\hline & $(0.0384)$ & $(0.0323)$ & $(0.0334)$ & $(0.0279)$ & $(0.0249)$ & $(0.0312)$ \\
\hline & [3.4151] & [4.8581] & [3.7453] & [5.6515] & [6.1714] & [4.4879] \\
\hline \multirow[t]{3}{*}{ D_RREQ(-3) } & $0.0105^{* *}$ & $0.0141^{* *}$ & $0.0098^{*}$ & $0.0259^{* * * *}$ & $0.0261^{* *}$ & $0.0343^{* *}$ \\
\hline & $(0.0049)$ & $(0.0053)$ & $(0.0058)$ & $(0.0085)$ & (0.0103) & (0.0149) \\
\hline & [2.1496] & [2.6119] & [1.6882] & [3.0593] & [2.5388] & [2.2907] \\
\hline \multirow[t]{3}{*}{ D_DEFAULT(-2) } & $0.8424^{* *}$ & $0.6502^{*}$ & $0.7319^{*}$ & $0.8978^{* *}$ & $0.7618^{* *}$ & $0.8973^{* *}$ \\
\hline & $(0.3535)$ & $(0.3702)$ & (0.4069) & $(0.4440)$ & $(0.3839)$ & $(0.4426)$ \\
\hline & [2.3832] & [1.7562] & [1.7984] & [2.0219] & [1.9846] & {$[2.0273]$} \\
\hline \multirow[t]{3}{*}{ D_EXCH(-2) } & & $3.3961^{* * *}$ & & & $3.9151^{* *}$ & \\
\hline & & $(1.2286)$ & & & (1.9323) & \\
\hline & & [2.7643] & & & {$[2.0261]$} & \\
\hline \multirow[t]{4}{*}{$\mathrm{D}_{-} \mathrm{IBOV}(-2)$} & & & $-0.0001^{* *}$ & & & $-0.0001^{* * *}$ \\
\hline & & & $(0.00004)$ & & & $(0.00003)$ \\
\hline & & & {$[-2.0496]$} & & & {$[-3.1621]$} \\
\hline & 0,9641 & 0,9671 & 0,9670 & 0,9471 & 0,9542 & 0,9471 \\
\hline Adjusted & 0,9628 & 0,9656 & 0,9655 & 0,9451 & 0,9520 & 0,9445 \\
\hline$F$-statistic & 745,1922 & 647,4386 & 645,0387 & & & \\
\hline Prob.(F-statistic) & 0,0000 & 0,0000 & 0,0000 & & & \\
\hline$J$-statistic & & & & 14,5610 & 12,7219 & 11,2616 \\
\hline Prob.( $J$-statistic $)$ & & & & 0,5570 & 0,6930 & 0,5889 \\
\hline Ramsey RESET (1) & 1,6230 & 1,2968 & 1,6473 & & & \\
\hline Prob.(Ramsey RESET) & 0,1075 & 0,1974 & 0,1024 & & & \\
\hline Heteroskedasticity ARCH (1) & 5,8658 & 1,7936 & 1,2550 & & & \\
\hline Prob.(Heteroskedasticity) & 0,0170 & 0,1832 & 0,2650 & & & \\
\hline Breusch-Godfrey - LM Test (1) & 10,4123 & 6,6495 & 6,3155 & & & \\
\hline Prob.(Breusch-Godfrey - LM Test) & 0,0016 & 0,0113 & 0,0134 & & & \\
\hline \multicolumn{7}{|l|}{ GMM - Instrumentos } \\
\hline \multicolumn{7}{|c|}{ Equação 7 - SPREAD(-2 to -4) SELIC(-2 to -8) D_RREQ(-4 to -10) D_DEFAULT2(-3 to -5) } \\
\hline \multicolumn{7}{|c|}{ Equação 8 - SPREAD(-2 to -3) SELIC(-2 to -8) D_RREQ(-4 to -9) D_DEFAULT2(-3 to -5$)$ D_EXCH( -3 to -5$)$} \\
\hline \multicolumn{7}{|c|}{ Equação 9 - SPREAD(-2) SELIC(-2 to -6) D_RREQ(-4 to -10) D_DEFAULT2(-3 to -6) D__BOV(-3) } \\
\hline \multicolumn{7}{|c|}{ Níveis de significância: ${ }^{* * *}$ denota $1 \%,{ }^{* *}$ denota $5 \%$ e *denota $10 \%$. Erros padrão entre parênteses e estatística $t$ entre colchetes. } \\
\hline
\end{tabular}




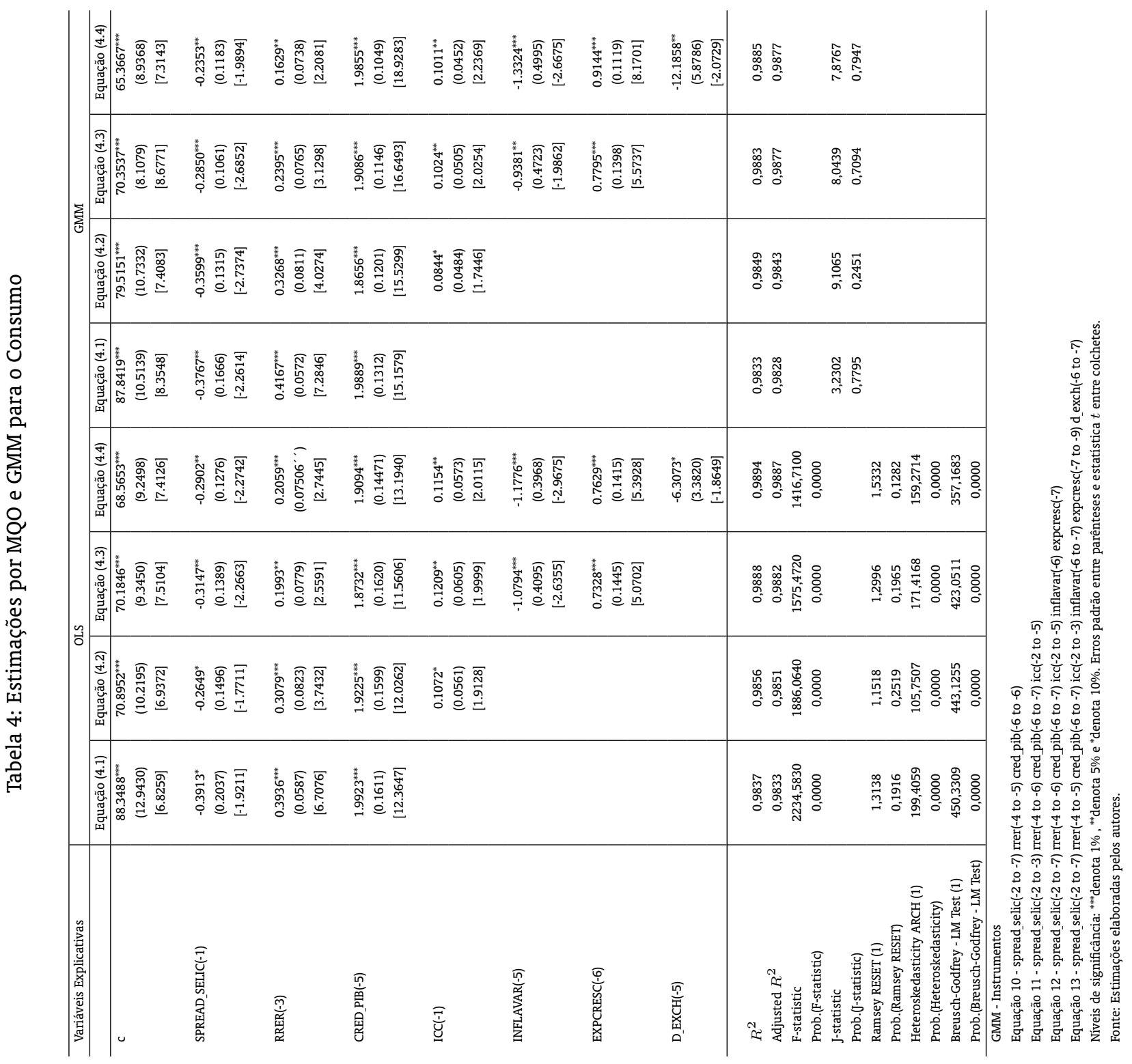




\subsection{Estimação do Consumo das Famílias}

A equação estimada para explicar o comportamento do consumo das famílias é explicitada abaixo (o sinal acima da variável refere-se ao sinal esperado da primeira derivada) e, $\rho_{t}$ representa o termo de erro.

$$
\begin{aligned}
\text { conssa } & =\varphi_{0}+\varphi_{1} \text { spread_selic }_{(-1)}^{(-)}+\varphi_{2} \operatorname{rrer}_{(-3)}^{(+)}+\varphi_{3} \operatorname{cred}_{\text {pib }}^{(+)}(+5)+\varphi_{4} i c c_{(-1)}^{(+)} \\
& +\varphi_{5} \text { inflavar }_{(-5)}^{(-)}+\varphi_{6} \exp \operatorname{cresc}_{(-6)}+\varphi_{7} \operatorname{dexch}_{(-5)}^{(-)}+\rho_{t}
\end{aligned}
$$

A Tabela 4 apresenta os resultados das estimativas por MQO e GMM.

Considerando os resultados da estimação da equação completa (4.4), todas as variáveis apresentam os sinais esperados e significância estatística, por MQO e GMM. As evidências indicam a existência de uma relação negativa entre o consumo das famílias e a taxa real de juros, o que corrobora as evidências existentes na literatura. Os resultados também apontam que aumentos da renda real e do volume de crédito em relação ao PIB afetam positivamente o consumo das famílias. Os resultados também indicam a existência de uma relação positiva entre o consumo das famílias e a confiança do consumidor, a existência de uma relação negativa entre o consumo das famílias e a variação positiva da taxa de inflação, de uma relação positiva entre o consumo das famílias e o comportamento da renda futura esperada (expectativa de crescimento do PIB), e de uma relação negativa entre o consumo das famílias e a taxa de câmbio.

\section{CONCLUSÃO}

O estudo analisou a influência da credibilidade e da reputação das autoridades monetária e fiscal sobre o comportamento da taxa básica de juros. Os resultados indicam que tanto a credibilidade quanto a reputação por parte das autoridades monetária e fiscal possuem relação negativa com o comportamento da taxa básica de juros. Ademais, a aceleração da inflação, o hiato do produto e uma pior administração da estrutura de indexação da dívida pública possuem uma relação negativa com o comportamento da taxa básica de juros.

A análise empírica também aponta que a taxa básica de juros e os requerimentos de reservas bancárias afetam positivamente o spread de crédito. Portanto, para o período analisado, os resultados sugerem a influência da política monetária sobre o spread de crédito na economia brasileira.

Os resultados obtidos também indicaram a existência de uma relação positiva entre o consumo das famílias e o comportamento da renda real, da renda futura esperada, da confiança do consumidor e do crédito, assim como uma relação negativa entre o consumo das famílias e o comportamento da taxa real de juros, da taxa de câmbio a da aceleração da inflação.

\section{BIBLIOGRAFIA}

Agénor, P. \& Taylor, M. P. (1993). Analysing credibility in high-inflation countries: A new approach. Economic Journal, 103:329-36.

Auel, M. C. \& de Mendonça, H. F. (2011). Macroeconomic relevance of credit channels: Evidence from an emerging economy under inflation targeting. Economic Modelling, 28:965-979.

Barro, R. J. \& Gordon, D. B. (1983). Rules, discretion and reputation in a model of monetary policy. Journal of Monetary Economics, 12:101-122.

Bernanke, B. \& Blinder, A. (1992). The federal funds rate and the channels of monetary transmission. American Economic Review, 82:901-921. 
Bernanke, B. \& Gertler, M. (1995). Inside the black box: The credit channel of monetary policy transmission. Journal of Economic Perspectives, 9:27-48.

Calvo, G. \& Guidotti, P. (1990). Indexation and maturity of government bonds: An exploratory model. In Dornbush, R. \& Draghi, M., editors, Public Debt Management: Theory and History. Cambridge University Press, Cambridge.

Cati, R. C., Garcia, M. G. P., \& Perron, P. (1999). Unit roots in the presence of abrupt governmental interventions with an application to Brazilian data. Journal of Applied Econometrics, 14:27-56.

Cecchetti, S. G. \& Krause, S. (2002). Central bank structure, policy efficiency and macroeconomic performance: Exploring empirical relationships. Review, Federal Reserve Bank of St. Louis, July-August:47-59.

Cragg, J. G. (1983). More efficient estimation in the presence of heteroscedasticity of unknown form. Econometrica, 51:751-763.

de Mendonça, H. F. (2007). Towards credibility from inflation targeting: The Brazilian experience. Applied Economics, 39:2599-2615.

de Mendonça, H. F. \& da Silva, R. T. (2008). Administração da dívida pública sob um regime de metas para inflação: Evidências para o caso brasileiro. Economia Aplicada, 12:635-657.

de Mendonça, H. F. \& de Guimarães e Souza, G. J. (2009). Inflation targeting credibility and reputation: The consequences for the interest rate. Economic Modelling, 26:1228-1238.

de Mendonça, H. F. \& Machado, M. R. (2013). Public debt management and credibility: Evidence from an emerging economy. Economic Modelling, 30:10-21.

Franses, P. H. \& Haldrup, N. (1994). The effects of additive outliers on tests for unit roots and cointegration. Journal of Business and Economic Statistics, 12:471-478.

Giavazzi, F. \& Missale, A. (2004). Public debt management in Brazil. NBER Working Paper 10.394, National Bureau of Economic Research, Cambridge, Massachusetts.

Giavazzi, F. \& Pagano, M. (1990). Confidence crises and public debt management. In Dornbush, R. \& Draghi, M., editors, Public Debt Management: Theory and History. Cambridge University Press, Cambridge.

Hansen, L. P. (1982). Large sample properties of generalized method of moments estimators. Econometrica, 50:1029-1054.

IMF - International Monetary Fund (2002). Assessing sustainability. Prepared by the Policy Development and Review Department. In consultation with the Fiscal Affairs, International Capital Markets, Monetary and Exchange Affairs, and Research Departments.

Kwiatkowski, D., Phillips, P. C. B., Schmidt, P., \& Shin, Y. (1992). Testing the null hypothesis of stationarity against the alternative of a unit root. Journal of Econometrics, 54:159-178.

Kydland, F. E. \& Prescott, E. C. (1977). Rules rather than discretion: The inconsistency of optimal plans. Journal of Political Economy, 85:473-492.

Mishkin, F. S. (2007). Monetary Policy Strategy. The MIT Press.

Montes, G. C. \& Bastos, J. c. A. (2014). Effects of reputation and credibility on monetary policy: Theory and evidence for Brazil. Journal of Economic Studies, 41. 
Montes, G. C. \& Machado, C. C. (2013). Credibility and the credit channel transmission of monetary policy: Theoretical model and econometric analysis for brazil. Journal of Economic Studies, 40:469492.

Montes, G. C. \& Machado, C. C. (2014). Efeitos da credibilidade e reputação sobre a taxa selic e a transmissão da política monetária para o investimento agregado pelo canal dos preços dos ativos. Pesquisa e Planejamento Econômico, 44:241-287.

Montes, G. C. \& Peixoto, G. (2014). Risk-taking channel, bank lending channel and the "paradox of credibility": Evidence from Brazil. Economic Modelling, forthcoming.

Wooldridge, J. M. (2001). Applications of generalized method of moments estimation. Journal of Economic Perspectives, 15:87-100. 


\section{A. APÊNDICE}

Tabela A.1 - Testes de Raiz Unitária (ADF)

\begin{tabular}{|c|c|c|c|c|c|c|c|}
\hline $\mathrm{ADF}$ & & & & & & & \\
\hline Série & lag length & test & Schwarz & $p$-value & $1 \%$ & $5 \%$ & $10 \%$ \\
\hline selic & 1 & $-5,12712$ & 2 & 0,0002 & $-4,037668$ & $-3,448348$ & $-3,149326$ \\
\hline inflavar & 11 & $-6,78936$ & 1 & 0,0000 & $-3,492523$ & $-2,888669$ & $-2,581313$ \\
\hline gap & 0 & $-4,50025$ & 3 & 0,0000 & $-2,584539$ & $-1,94354$ & $-1,614941$ \\
\hline icbc & 0 & $-3,88071$ & 1 & 0,0029 & $-3,486064$ & $-2,885863$ & $-2,579818$ \\
\hline irm & 2 & $-4,72885$ & 1 & 0,0001 & $-3,487046$ & $-2,88629$ & $-2,580046$ \\
\hline $\operatorname{irf}$ & 0 & $-3,37754$ & 1 & 0,0137 & $-3,486064$ & $-2,885863$ & $-2,579818$ \\
\hline icf & 2 & $-1,82071$ & 1 & 0,3689 & $-3,487046$ & $-2,88629$ & $-2,580046$ \\
\hline d_icf & 1 & $-2,27097$ & 3 & 0,0229 & $-2,584877$ & $-1,943587$ & $-1,614912$ \\
\hline index & 6 & $-2,18909$ & 3 & 0,0281 & $-2,585587$ & $-1,943688$ & $-1,61485$ \\
\hline d_Index & 5 & $-5,73117$ & 3 & 0,0000 & $-2,585587$ & $-1,943688$ & $-1,61485$ \\
\hline prz & 0 & $-2,45847$ & 2 & 0,3481 & $-4,036983$ & $-3,448021$ & $-3,149135$ \\
\hline d_prz & 0 & $-11,99129$ & 2 & 0,0000 & $-4,037668$ & $-3,448348$ & $-3,149326$ \\
\hline spread & 1 & $-1,60184$ & 3 & 0,1026 & $-2,584707$ & $-1,943563$ & $-1,614927$ \\
\hline rreq & 1 & $-0,82731$ & 3 & 0,3556 & $-2,584707$ & $-1,943563$ & $-1,614927$ \\
\hline $\mathrm{d}_{-}$rreq & 0 & $-8,90217$ & 2 & 0,0000 & $-4,037668$ & $-3,448348$ & $-3,149326$ \\
\hline default & 3 & $-0,22259$ & 3 & 0,6041 & $-2,58505$ & $-1,943612$ & $-1,614897$ \\
\hline d_default & 2 & $-3,22359$ & 3 & 0,0015 & $-2,58505$ & $-1,943612$ & $-1,614897$ \\
\hline ibov & 1 & $-2,23911$ & 2 & 0,4634 & $-4,037668$ & $-3,448348$ & $-3,149326$ \\
\hline dibov & 0 & $-8,97632$ & 2 & 0,0000 & $-4,037668$ & $-3,448348$ & $-3,149326$ \\
\hline exch & 1 & $-3,35425$ & 1 & 0,0146 & $-3,486551$ & $-2,886074$ & $-2,579931$ \\
\hline d_exch & 0 & $-7,52866$ & 3 & 0,0000 & $-2,584707$ & $-1,943563$ & $-1,614927$ \\
\hline conssa & 1 & $-3,99520$ & 2 & 0,0113 & $-4,037668$ & $-3,448348$ & $-3,149326$ \\
\hline spread_selic & 1 & $-3,15331$ & 2 & 0,0991 & $-4,037668$ & $-3,448348$ & $-3,149326$ \\
\hline rrer & 1 & $-1,80445$ & 3 & 0,0678 & $-2,584707$ & $-1,943563$ & $-1,614927$ \\
\hline cred pib & 1 & $-3,35286$ & 2 & 0,0630 & $-4,037668$ & $-3,448348$ & $-3,149326$ \\
\hline expcresc & 7 & $-4,50602$ & 1 & 0,0003 & $-3,489659$ & $-2,887425$ & $-2,580651$ \\
\hline icc & 0 & $-3,40645$ & 2 & 0,0554 & $-4,036983$ & $-3,448021$ & $-3,149135$ \\
\hline
\end{tabular}

(1) intercept - (2) trend and intercept - (3) none - Critério de Schwarz

Lag Length: Automatic - based on SIC, maxlag $=12$.

Fonte: Elaboração dos autores. 
Tabela A.2 - Testes de Raiz Unitária (PP)

\begin{tabular}{l|c|c|c|c|c|c|c}
\hline PP & & & & & & & \\
\hline Série & Bandwidth & test & Schwarz & p-value & $1 \%$ & $5 \%$ & $10 \%$ \\
\hline selic & 8 & $-2,19066$ & 3 & 0,0280 & $-2,584539$ & $-1,94354$ & $-1,614941$ \\
inflavar & 3 & $-5,47519$ & 2 & 0,0001 & $-4,037668$ & $-3,448348$ & $-3,149326$ \\
gap & 4 & $-4,52842$ & 3 & 0,0000 & $-2,584539$ & $-1,94354$ & $-1,614941$ \\
icbc & 5 & $-3,75965$ & 1 & 0,0043 & $-3,486064$ & $-2,885863$ & $-2,579818$ \\
irm & 5 & $-2,67342$ & 1 & 0,0817 & $-3,486064$ & $-2,885863$ & $-2,579818$ \\
irf & 8 & $-3,44953$ & 1 & 0,0111 & $-3,486064$ & $-2,885863$ & $-2,579818$ \\
icf & 8 & $-0,36234$ & 2 & 0,9879 & $-4,036983$ & $-3,448021$ & $-3,149135$ \\
d_icf & 5 & $-2,11511$ & 3 & 0,0336 & $-2,584707$ & $-1,943563$ & $-1,614927$ \\
index & 4 & $-9,92002$ & 1 & 0,0000 & $-3,486064$ & $-2,885863$ & $-2,579818$ \\
d_Index & 8 & $-9,92596$ & 2 & 0,0000 & $-4,037668$ & $-3,448348$ & $-3,149326$ \\
prz & 10 & 1,75441 & 3 & 0,9806 & $-2,584539$ & $-1,94354$ & $-1,614941$ \\
d_prz & 11 & $-11,98695$ & 2 & 0,0000 & $-4,037668$ & $-3,448348$ & $-3,149326$ \\
spread & 3 & $-1,63563$ & 3 & 0,0960 & $-2,584539$ & $-1,94354$ & $-1,614941$ \\
rreq & 2 & $-0,76803$ & 3 & 0,3816 & $-2,584539$ & $-1,94354$ & $-1,614941$ \\
d_rreq & 1 & $-8,93449$ & 3 & 0,0000 & $-2,584707$ & $-1,943563$ & $-1,614927$ \\
default & 9 & $-2,14894$ & 2 & 0,5131 & $-4,036983$ & $-3,448021$ & $-3,149135$ \\
d_default & 7 & $-10,28760$ & 3 & 0,0000 & $-2,584707$ & $-1,943563$ & $-1,614927$ \\
ibov & 5 & $-2,20344$ & 2 & 0,4830 & $-4,036983$ & $-3,448021$ & $-3,149135$ \\
d_ibov & 4 & $-8,93080$ & 3 & 0,0000 & $-2,584707$ & $-1,943563$ & $-1,614927$ \\
exch & 1 & $-2,68671$ & 1 & 0,0793 & $-3,486064$ & $-2,885863$ & $-2,579818$ \\
d_exch & 3 & $-7,69423$ & 3 & 0,0000 & $-2,584707$ & $-1,943563$ & $-1,614927$ \\
conssa & 5 & $-3,14066$ & 2 & 0,1019 & $-4,036983$ & $-3,448021$ & $-3,149135$ \\
spread_selic & 1 & $-1,56047$ & 3 & 0,1112 & $-2,584539$ & $-1,94354$ & $-1,614941$ \\
rrer & 5 & $-2,00498$ & 3 & 0,0435 & $-2,584539$ & $-1,94354$ & $-1,614941$ \\
cred_pib & 4 & $-3,49060$ & 2 & 0,0450 & $-4,036983$ & $-3,448021$ & $-3,149135$ \\
expcresc & 6 & $-1,24513$ & 3 & 0,1950 & $-2,584539$ & $-1,94354$ & $-1,614941$ \\
icc & 2 & $-3,60846$ & 2 & 0,0333 & $-4,036983$ & $-3,448021$ & $-3,149135$ \\
\hline
\end{tabular}

(1) intercept - (2) trend and intercept - (3) none - Critério de Schwarz A defasagem é o lag truncation escolhido para o Bartlett kernel. Fonte: Elaboração dos autores. 
Tabela A.3 - Testes de Estacionariedade (KPSS)

\begin{tabular}{l|c|c|c|c|c|c}
\hline KPSS & & & & & & \\
\hline Série & Bandwidth & LM test & Schwarz & $1 \%$ & $5 \%$ & $10 \%$ \\
\hline selic & 9 & 0,144422 & 2 & 0,216 & 0,146 & 0,119 \\
inflavar & 6 & 0,050765 & 2 & 0,216 & 0,146 & 0,119 \\
gap & 8 & 0,044606 & 1 & 0,739 & 0,463 & 0,347 \\
icbc & 8 & 0,303685 & 1 & 0,739 & 0,463 & 0,347 \\
irm & 8 & 0,178763 & 2 & 0,216 & 0,146 & 0,119 \\
irf & 9 & 0,118968 & 2 & 0,216 & 0,146 & 0,119 \\
icf & 9 & 0,255192 & 2 & 0,216 & 0,146 & 0,119 \\
dicf & 3 & 0,185866 & 1 & 0,739 & 0,463 & 0,347 \\
index & 9 & 0,272163 & 2 & 0,216 & 0,146 & 0,119 \\
d_Index & 8 & 0,214746 & 2 & 0,216 & 0,146 & 0,119 \\
prz & 9 & 0,150288 & 2 & 0,216 & 0,146 & 0,119 \\
d_prz & 9 & 0,295669 & 1 & 0,739 & 0,463 & 0,347 \\
spread & 8 & 0,108064 & 2 & 0,216 & 0,146 & 0,119 \\
rreq & 9 & 0,202371 & 1 & 0,739 & 0,463 & 0,347 \\
d_rreq & 2 & 0,117043 & 1 & 0,739 & 0,463 & 0,347 \\
default & 9 & 0,066600 & 2 & 0,216 & 0,146 & 0,119 \\
d_default & 7 & 0,097939 & 1 & 0,739 & 0,463 & 0,347 \\
ibov & 9 & 0,192211 & 2 & 0,216 & 0,146 & 0,119 \\
dibov & 4 & 0,105371 & 1 & 0,739 & 0,463 & 0,347 \\
exch & 8 & 0,274182 & 2 & 0,216 & 0,146 & 0,119 \\
d_exch & 9 & 0,033840 & 2 & 0,216 & 0,146 & 0,119 \\
conssa & 0,208692 & 2 & 0,216 & 0,146 & 0,119 \\
spread_selic & 9 & 0,059532 & 2 & 0,216 & 0,146 & 0,119 \\
rrer & 0,197267 & 2 & 0,216 & 0,146 & 0,119 \\
cred pib & 0,166691 & 2 & 0,216 & 0,146 & 0,119 \\
expcresc & 0,114808 & 1 & 0,739 & 0,463 & 0,347 \\
icc & 0,059702 & 2 & 0,216 & 0,146 & 0,119 \\
\hline icinter & 9 & 2 & 2 & & \\
\hline
\end{tabular}

(1) intercept - (2) trend and intercept - Critério de Schwarz

A defasagem é o lag truncation escolhido para o Bartlett kernel. Fonte: Elaboração dos autores. 